\title{
Identification of Diatoms by Grid Graph Matching
}

\author{
Stefan Fischer, Kaspar Gilomen, and Horst Bunke \\ Institute of Computer Science and Applied Mathematics \\ University of Bern, Switzerland \\ \{fischer, bunke\}@iam.unibe.ch
}

\begin{abstract}
Diatoms are unicellular algae found in water and other places wherever there is humidity and enough light for photo synthesis. In this paper a graph matching based identification approach for the retrieval of diatoms from an image database is presented. The retrieval is based on the matching of labeled grid graphs carrying texture information of the underlying diatom. A grid graph is a regular, rectangular arrangement of nodes overlaid on an image. Each node of the graph is labeled with texture features describing a rectangular sub-region of the object. Properties of gray level co-occurrence matrices as well as of Gabor wavelets are used as texture features. The method has been evaluated on a diatom database holding images of 188 different diatoms belonging to 38 classes. For the identification of these diatoms recognition rates of more than 90 percent were obtained.
\end{abstract}

\section{Introduction}

In this paper an approach to the identification of diatoms based on the matching of labeled grid graphs is presented. The work has been done in the framework of the ADIAC project [1] which aims at the automatic identification and classification of diatoms. Diatoms are unicellular algae found in water and other places wherever there is humidity and enough light for photo synthesis. Diatom identification has a number of applications in areas such as environmental monitoring, climate research and forensic medicine [14]. One of the great challenges in diatom identification is the large number of classes involved ${ }^{1}$. Experts estimate the number of diatom species between 15000 and 20000, or even higher. Diatoms are characterized by an ornamented cell wall composed of silica, which is highly resistant and remains after chemical cleaning where all organic contents are removed. The cell wall consist of two valves that fit within each other like the pieces of a petri dish. The identification as well as the taxonomy of diatoms is based on the morphology of these silica valves. Example images of valves of four different classes of diatoms are shown in Figure 1. As can be seen diatoms

\footnotetext{
${ }^{1}$ In terms of biologists diatoms are hierarchically classified in genus, species, subspecies and so forth, but in this paper we'll use the term class in the pattern recognition sense.
} 


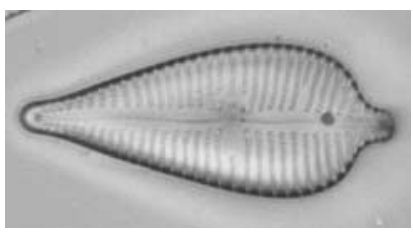

a.)

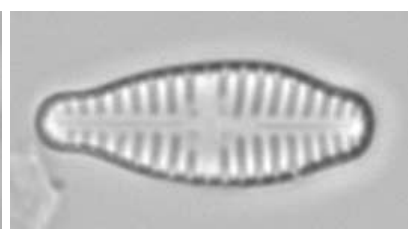

b.)

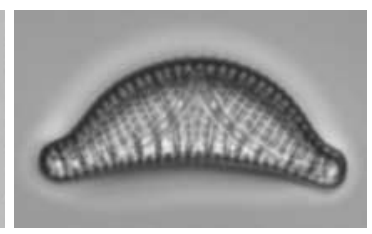

c.)

Fig. 1. Example images of diatom valves in face view (top-down view): a.) Gomphonema augur var. augur (Ehrenberg), b.) Gomphonema olivaceum (Möller), c.) Epithemia sorex var. sorex (Kützing)

are of different shape and morphological structure. Furthermore, for some diatoms the size and the shape of the valve changes during the live cycle, but the morphology of the valve keeps mostly the same. While in previous studies the variation of the shape and shape based identification have been studied ([4], [5], [10], [11], [13], [17]), the objective of the present paper is the identification of diatoms based on the morphology of the valve.

In the retrieval approach presented here, texture features of the morphological structure of diatom valves are extracted and taken as node labels of a grid graph. Grid graphs are a special class of graphs which correspond to a regular, rectangular arrangement of nodes overlaid on an image. In Figure 2 an example of a grid graph is shown. The inner nodes of the graph are connected with their four neighbors in horizontal and vertical direction. The outer nodes are connected with two or three neighbors, depending whether they correspond to a corner or not. For the diatom images stored in the reference image database, labeled grid-graphs are pre-computed and attached to each image. During the retrieval phase the similarity between the query image and images in the database is evaluated by matching the corresponding grid graphs. In this phase the query image is overlaid with a grid and texture properties are computed in image regions surrounding the nodes. To take the changing size of diatoms into regard, the position of the nodes is varied. A distance measure between the different graphs extracted from the query image and all graphs stored in the database is computed. As result of the query the most similar images found in the database are returned to the user.

The remainder of the paper is organized as follows. In Section 2 a short overview of grid graph matching techniques found in other pattern recognition applications will be given. The representation of objects, in our case diatoms, will be described in Section 3. The matching procedure will be outlined in Section 4. Experimental results will be reported in Section 5 and conclusions drawn in Section 6. 


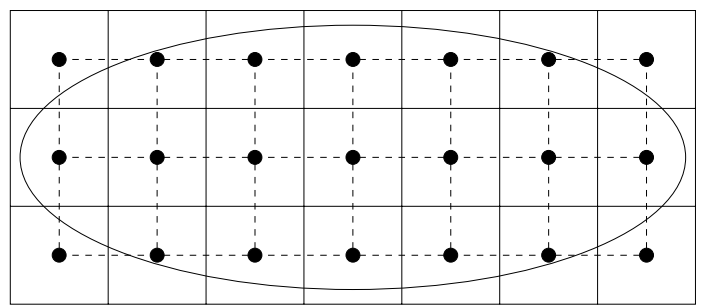

Fig. 2. Grid graph with $3 \times 7$ nodes representing the underlying object outlined by an ellipse

\section{Related Work in Grid Graph Matching}

Labeled graph matching has been successfully used in numerous object recognition tasks [3]. Grid graphs are a special subclass of graphs. Most applications of grid graph matching are focused on face recognition tasks such as the detection, tracking, or identification of persons ([9], [15], [18]). There are two main sources of information which are used for face recognition based on grid graph matching. One source is geometrical features, for example, the position of the nose, mouth and eyes. The second source is gray level and texture information of the skin. An approach that exploits both sources of information is the so-called dynamic link architecture [9]. This approach is divided into a training and a recall phase. In the training phase, a sparse grid is build for each person in the reference database. The grid is overlaid on the facial region of a person's image and the response of a set of Gabor filters is measured at the grid nodes. The Gabor filters are tuned to different orientations and scales. In the recall phase, the reference grid of each person is overlaid on the face image of a test person and is deformed so that a cost function is minimized. The cost function is based on the differences between the feature vectors stored at the nodes of the reference grids and the feature vectors computed at certain pixel coordinates in the test image. Additionally, costs for the distortion between the reference grid and the variable graph built on the image of the test person are taken into account. The cost function is a measure of similarity of the model grid graph to the test graph. Similar ideas are used in the grid graph matching technique proposed in this paper.

\section{Object Representation by Means of Grid Graphs}

An important characteristic used in the identification of diatoms is the morphology of the valve face. In this paper we propose a complementary approach based on texture measures computed in local image regions. The identification of texture has been extensively studied in the computer vision literature. There are statistical methods that measure variance, entropy or energy of the gray level distribution of an image. Moreover, perceptual techniques have been proposed, which 


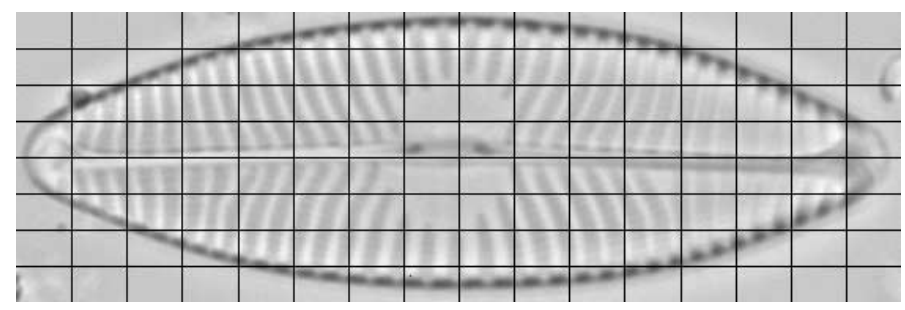

Fig. 3. Example image of windowing with a grid of dimension $16 \times 8$

are able to identify the direction, orientation and regularity of textures [12]. Among the most widely used texture measures are those derived from gray level co-occurrence matrices [7], and features based on Gabor wavelets [8,16]. These features have been adopted in the system described in this paper.

From the general point of view, almost any visual pattern can be represented via a graph containing nodes labeled with local features and links encoding the spatial relationship between the features [2]. It can be observed that many diatom valves consist of areas having relatively homogeneous texture. Thus, a diatom can be divided into separate areas and the texture can be measured in each area. The spatial relationships between such areas are preserved by overlaying a grid on the object. This is visualized in Figure 3. The example image is overlaid by a grid of $16 \times 8$ rectangular regions. The morphology of the valve face is then described by average values of characteristic properties inside these rectangular regions. As such properties, 13 features of the gray level co-occurrence matrix and the mean and standard deviation of 4 Gabor functions with different orientation are used. The features of each rectangular region are assigned as an attribute vector to the corresponding node of the grid graph as shown in Figure 2. For further details about the 13 textural features used as attributes of the grid graphs, the reader is referred to [6].

The textural features as well as the grid graph matching approach described in the next section are not invariant w.r.t object rotation. From the theoretical point of view, the development of an invariant recognition procedure would be very interesting. However the actual application doesn't require for rotational invariance because the images of both the diatoms in the database and the query diatoms are acquired by a human operator, who manually aligns the objects such that they appear in a standard pose. Diatom image acquisition is quite time consuming. Hence this manual alignment doesn't add any significant overhead to the overall retrieval process. (The issue of scale invariance will be addressed in Section 4.)

\section{Grid Graph Matching}

Based on the grid graph representation, the problem of diatom identification can be formulated as a labeled graph matching task, where the goal is to find 
an optimal one-to-one correspondence between the nodes of an input graph and the nodes of a graph stored in the database. A good correspondence is one that respects the spatial relationships between the nodes, and exhibits a high degree of similarity between the labels of the corresponding nodes $[2,3]$.

In our implementation, the dissimilarity of two grid graphs is measured as the sum of distances of the nodes of two grid graphs. That is, the distance $\delta\left(G_{1}, G_{2}\right)$ between two graphs $G_{1}=\left(V_{1}, E_{1}\right)$ and $G_{2}=\left(V_{2}, E_{2}\right)$, where $V_{i}$ and $E_{i}$ denotes the set of nodes and edges of graph $G_{i}$, respectively, is defined as:

$$
\delta\left(G_{1}, G_{2}\right)=\frac{1}{\left|M\left(V_{1}, V_{2}\right)\right|} \sum_{\left(v_{i}, v_{j}\right) \in M\left(V_{1}, V_{2}\right)} d\left(v_{i}, v_{j}\right) .
$$

The quantity $d\left(v_{i}, v_{j}\right)$ denotes the distance of feature vectors of a pair of nodes (see Eqn. (2)), and $M\left(V_{1}, V_{2}\right)$ is the set of all pairs of nodes $v_{i} \in V_{1}$ and $v_{j} \in V_{2}$ with similar spatial positions. This means if $p_{i}$ is the position of the node $v_{i}$ and $p_{j}$ is the position of the node $v_{j}$, then the constraint $\left|p_{i}-p_{j}\right|<\epsilon$ is fulfilled for each pair $\left(v_{i}, v_{j}\right)$ in $M$. To gain size invariance, the node positions are coded as distances relative to the center of the bounding box of the object.

In the simple version of the grid graph matching approach no distortion of the nodes' position is allowed. Thus, the above constraint becomes $p_{i}=p_{j}$. In this case the distance between two nodes $v_{i} \in V_{1}$ and $v_{j} \in V_{2}$ is defined as the distances between the feature vectors

$$
d\left(v_{i}, v_{j}\right)=\frac{1}{N} \sum_{n=1}^{N}\left|\mathrm{f}_{i, n}-\mathrm{f}_{j, n}\right|
$$

where $\mathrm{f}_{i, n}$ is the $n$-th feature of the node $v_{i}$ and $N$ is the number of features in the feature vectors. As the ranges of the various features are different they are normalized in a pre-processing step. In our approach the min-max normalization is used to normalize all feature values to the interval $[0,1]$. For each feature $f$ the minimum $\mathrm{f}_{\min }$ and the maximum $\mathrm{f}_{\max }$ over the training set are computed and the normalized feature value $\mathrm{f}^{\prime}$ is calculated as

$$
\mathrm{f}^{\prime}=\frac{\mathrm{f}-\mathrm{f}_{\min }}{\mathrm{f}_{\max }-\mathrm{f}_{\min }} .
$$

Whenever a feature of a query diatom has a value smaller than $\mathrm{f}_{\text {min }}$, or larger than $\mathrm{f}_{\max }$, the normalized value is set equal to 0 , or 1 , respectively.

The grid graph distance defined by Eqn. (1) is sensitive to geometric distortions. In order to improve its robustness, a second grid graph distance, called flexible grid graph distance, is introduced. In flexible grid graph matching, each node of the query graph can be translated, by a small degree, in the image plane. Note that the nodes of the database graph remain fixed.

In the flexible grid graph distance an additional cost is introduced for each node, indicating whether a translation is applied to the node or not. If no translation is applied this additional cost is equal to zero. Formally, let the translation cost be defined as

$$
t\left(v_{i}, v_{j}\right)=t\left(v_{j}\right)
$$


where $v_{i}$ belongs to a graph from the database and $v_{j}$ to a query graph. Moreover,

$$
t(v)=\left\{\begin{array}{ll}
c & \text { if } v \text { has been translated } \\
0 & \text { otherwise }
\end{array} .\right.
$$

Using this additional term in the cost function we define the flexible node distance as (cf. Eqn. (2))

$$
d^{\prime}\left(v_{i}, v_{j}\right)=\min _{T}\left[\frac{1}{N} \sum_{n=1}^{N}\left(\left|\mathrm{f}_{i, l}-\mathrm{f}_{j, l}\right|\right)+t\left(v_{i}, v_{j}\right)\right]
$$

In this equation, $T$ is the set of all translations that can be applied to the nodes. The aim of each translation is to reduce the matching cost of the two feature vectors, i.e. to make $\sum\left(\left|\mathrm{f}_{i, l}-\mathrm{f}_{j, l}\right|\right)$ smaller. However, because there is a cost for each translation, a trade-off between the cost resulting from the feature vector difference and from the node translations arises. In the flexible distance $d^{\prime}\left(v_{i}, v_{j}\right)$ this cost is minimized over all possible translations of the nodes. The parameter $c$ is the same for all nodes and has to be chosen empirically. Theoretically, a more general model could be adopted, where $t(v)$ depends on the actual degree of translation. This generalization, however, results in many more free parameters in the cost function that need to be defined. To avoid this problem a constant cost of node translations has been used.

Given the flexible distance of a pair of nodes defined by Eqn. (6), the flexible grid graph distance $\delta^{\prime}$ is defined as follows

$$
\delta^{\prime}\left(G_{1}, G_{2}\right)=\frac{1}{\left|M\left(V_{1}, V_{2}\right)\right|} \sum_{\left(v_{i}, v_{j}\right) \in M\left(V_{1}, V_{2}\right)} d^{\prime}\left(v_{i}, v_{j}\right) .
$$

\section{Experimental Results}

The proposed grid graph matching based identification approach for the retrieval of diatoms is evaluated on a test database of 188 images of different diatoms. The diatoms belong to 38 different classes. In the Appendix a sample image for each class can be found.

In the test database, most of the classes are only represented by 3 to 6 images. Actually, the minimum and maximum number of representatives is 3 and 9 images, respectively. Because of this limited number of images the performance of the approach is validated using the leave-one-out technique. This means each sample in the databases is once used for testing and all other samples are used as prototypes. This procedure is repeated until each sample is used exactly once for testing.

In a first test the standard grid graph matching procedure is used, and in the second test the flexible matching is applied. The results of the first test are visualized in Figure 4. The recognition rate achieved using only features of the gray level co-occurrence matrix are drawn as a dashed line, the rate using features of Gabor wavelets as dotted line, and the combination of both sets of 


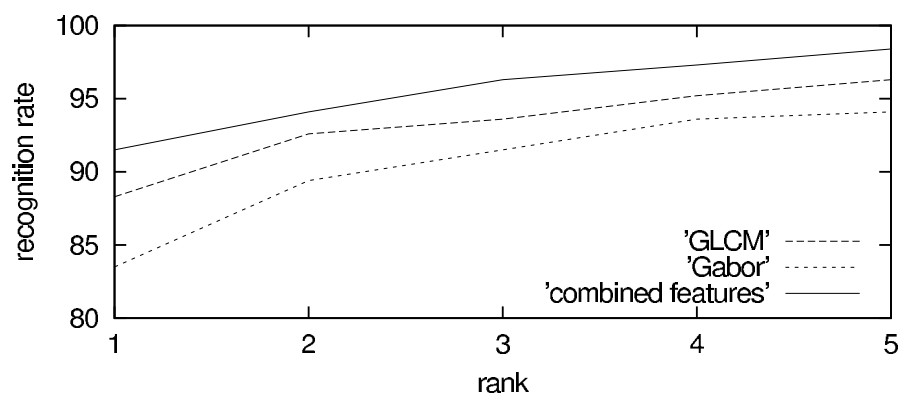

Fig. 4. Results for identification of diatoms using standard grid graph matching

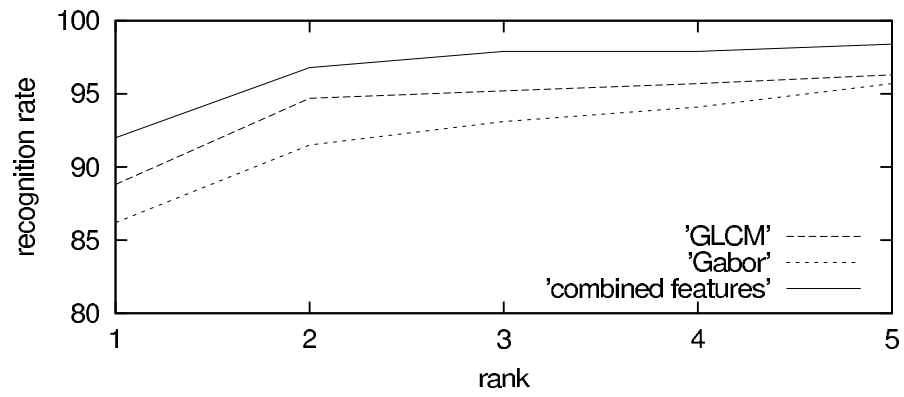

Fig. 5. Results for identification of diatoms using flexible grid graph matching

features as solid line. On the y-axis the recognition rate is given and on the $\mathrm{x}$-axis the highest rank taken into regard. Thus, for example, rank 2 in the chart represents the accumulated recognition rate for all samples whose real class is detected as the most, or second most, similar image by the matching procedure. As can be seen in Figure 4 the recognition rate using only features of the gray level co-occurrence matrix is slightly higher than the recognition rate obtained by features of the Gabor wavelets. The highest recognition rate is obtained by combining both feature sets.

In the second test the flexible grid graph matching is used which allows distortions of the nodes. As can be seen in Figure 5 the recognition rates for all three feature sets are higher than in the previous test. Especially when including the second rank better results are obtained. Instead of $94 \%$ in Fig. 4 now nearly $97 \%$ are obtained. Thus, if a set of similar images is returned for a query image the probability that images of the same class are in the result set is higher for flexible grid graph matching then for the simple matching approach. 


\section{Conclusion}

In this paper we have proposed a flexible grid graph matching based identification approach for the retrieval of diatoms from an image database. The application of graph matching methods for the identification of diatoms turned out to be especially useful for the description of morphological properties, which change during the live cycle of diatoms. As features texture properties of gray level co-occurrence matrices and Gabor wavelets of local image regions were used.

On a complex database holding images of 38 different classes of diatoms recognition rates of nearly $98 \%$ were achieved if the first three ranks are taken into regard. These rates are impressive regarding the difficulty of the considered task. As one can conclude from the images shown in the Appendix, some classes are quite similar in shape, and others have a similar valve structure. Another complication arises from the small size of the available database. There are individuals in some classes that are quite different from the individuals in the same class. These outliers are very prone to being misclassified by the selected classification procedure.

The system described in this paper is a small prototype that was built to study the feasibility of grid graph matching for automatic identification and retrieval. The recognition rate of $98 \%$, considering the first three ranks, seems very promising for real application of the system. In future versions of the system it is planed to significantly increase the number of diatom classes. From such an increase, a drop of the recognition performance must be expected. However, in the context of ADIAC [1] several other methods for diatom identification are under development. They are based on different features, for example, shape and global texture features, and different recognition procedures, for example, decision tree based classification. These methods have characteristics that are quite complementary to the approaches described in this paper. Therefore, it can be expected that the combination of these methods with the grid graph matching approach proposed in this paper will further improve the recognition rate. Such a combination will be one of our future research topics.

\section{Acknowledgment}

The work has been done in the framework of the EU-sponsored Marine Science and Technology Program (MAST-III), under contract no. MAS3-CT97-0122. Additional funding came from the Swiss Federal Office for Education and Science (BBW 98.00.48). We thank our project partners Micha Bayer and Stephen Droop from Royal Botanic Garden Edinburgh and Steve Juggins and co-workers at Newcastle University for preparing the images in the ADIAC image database and for useful discussions and hints. 


\section{References}

1. Automatic Diatom Identification And Classification. Project home page: http://www.ualg.pt/adiac/. 94, 101

2. E. Bienenstock and C. von der Malsburg. A neural network for invariant pattern recognition. Europhysics Letters, 4:121-126, 1987. 97, 98

3. H. Bunke. Recent developments in graph matching. In Proceedings of the 15th International Conference on Pattern Recognition (ICPR '00), volume 2, pages 117124, Barcelona, Spain, September 3-8 2000. 96, 98

4. S. Fischer, M. Binkert, and H. Bunke. Feature based retrieval of diatoms in an image database using decision trees. In Proceedings of the 2nd International Conference on Advanced Concepts for Intelligent Vision Systems (ACIVS 2000), pages 67-72, Baden-Baden, Germany, August 2000. 95

5. S. Fischer, M. Binkert, and H. Bunke. Symmetry based indexing of diatoms in an image database. In Proceedings of the 15th International Conference on Pattern Recognition (ICPR '00), volume 2, pages 899-902, Barcelona, Spain, September 3-8 2000. 95

6. K. Gilomen. Texture based identification of diatoms (in German). Master's thesis, University of Bern, 2001. 97

7. R. M. Haralick, K. Shanmugam, and I. Dinstein. Textural features for image classification. IEEE Transactions on Systems, Man, and Cybernetics, pages 610621, 1973. 97

8. A. K. Jain and F. Farrokhnia. Unsupervised texture segmentation using Gabor filters. Pattern Recognition, 24(12):1167-1186, 1991. 97

9. M. Lades, J. Vorbrüggen, J. Buhmann, J. Lange, C. von der Malsburg, R. Würtz, and W. Konen. Distortion invariant object recognition in the dynamic link architecture. IEEE Transaction on Computers, 42(3):300-311, 1993. 96

10. D. Mou and E. F. Stoermer. Separating tabellaria (bacillariophyceae) shape groups: A large sample approach based on fourier descriptor analysis. Journal of Phycology, 28:386-395, 1992. 95

11. J. L. Pappas and E. F. Stoermer. Multidimensional analysis of diatom morphological phenotypic variation and relation to niche. Ecoscience, 2:357-367, 1995. 95

12. I. Pitas. Digital image processing algorithms. Prentice Hall, London, 1993. 97

13. E. F. Stoermer. A simple, but useful, application of image analysis. Journal of Paleolimnology, 15:111-113, 1996. 95

14. E. F. Stoermer and J. P. Smol, editors. The Diatoms: Applications for the Environmental and Earth Science. Cambridge University Press, 1999. 94

15. A. Tefas, C. Kotropoulos, and I. Pitas. Using support vector machines to enhance the performance of elastic graph matching for frontal face authentication. IEEE Transactions on Pattern Analysis and Machine Intelligence, 23:735-746, 2001. 96

16. M. Tuceryan and A. K. Jain. Texture analysis. In C. H. Chen, L. F. Pau, and P. S. P. Wang, editors, The Handbook of Pattern Recognition and Computer Vision, pages 207-248. World Scientific Publishing Co, 2 edition, 1998. 97

17. M. Wilkinson, J. Roerdink, S. Droop, and M. Bayer. Diatom contour analysis using morphological curvature scale spaces. In Proceedings of the 15th International Conference on Pattern Recognition (ICPR '00), pages 656-659, Barcelona, Spain, September 3-7 2000. 95

18. L. Wiskott, J. Fellous, N. Krüger, and C. von der Malsburg. Face recognition by elastic bunch graph matching. IEEE Transactions on Pattern Analysis and Machine Intelligence, 19(7):775-779, 1997. 96 


\section{Appendix}

For more images see: http://www.ualg.pt/adiac/.

Table 1. Example images of the classes 1 to 38 included in the test database

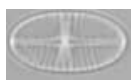

Achnanthes saxonica Amphora pediculus

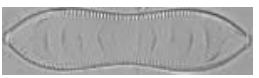

Cymatopleura solea

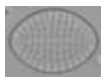

Delphineis minutissima Diatoma vulgaris
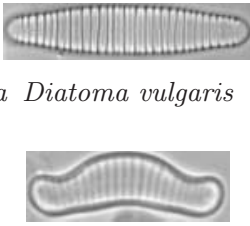

Eunotia exigua

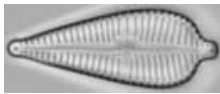

Gomphonema augur
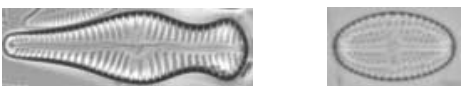

Gomphonema truncatum Luticola mutica

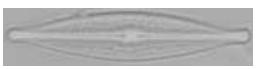

Brachysira vitrea

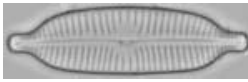

Cymbella hybrida

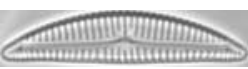

Encyonema neogracile

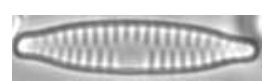

Fragilaria vaucheriae Fragilaria virescens

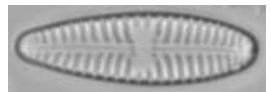

Gomphonema olivaceum

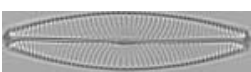

Navicula lanceolata

Navicula palpebralis

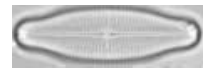

Navicula protracta

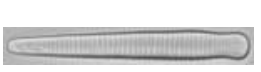

Peronia fibula

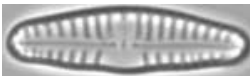

Reimeria sinuata

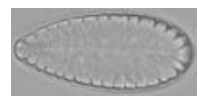

Surirella brebissonii Tabellaria flocculosa
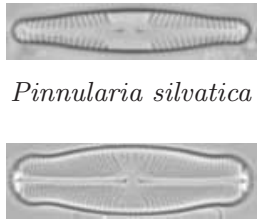

Sellaphora pupula

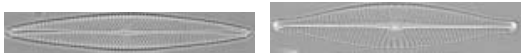

Navicula radiosa Navicula rhynchocephala Nitzschia recta

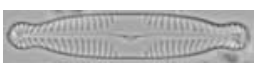

Pinnularia silvatica Pinnularia subcapitata

Pinnularia viridi
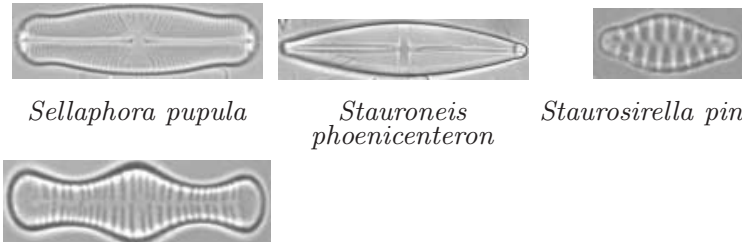

Stauroneis phoenicenteron 